\title{
Ground and Excited States of Singlet, Cation Doublet, and Anion Doublet States of $o$-Benzoquinone: A Theoretical Study
}

\author{
Yasushi Honda, ${ }^{*, \dagger, \ddagger}$ Masahiko Hada, ${ }^{\dagger, *}$ Masahiro Ehara, ${ }^{\S}$ and Hiroshi Nakatsuji ${ }^{\S}$ \\ Department of Chemistry, Graduate School of Science and Engineering, Tokyo Metropolitan University, \\ 1-1 Minami-Osawa, Hachioji-shi, Tokyo 192-0397, Japan, CREST, Japan Science and Technology (JST) \\ Agency, and Department of Synthetic Chemistry and Biological Chemistry, Graduate School of Engineering, \\ Kyoto University, Nishikyo-ku, Kyoto 615-8510, Japan
}

Received: December 16, 2006; In Final Form: February 6, 2007

\begin{abstract}
The singlet excited states and doublet ionized/electron-attached states of $o$-benzoquinone (o-BQ) were studied by the symmetry adapted cluster (SAC)/ SAC-configuration interaction (SAC-CI) method, and the corresponding spectral bands were assigned. The calculated transition energies reasonably reproduced the experimental spectra with the mean error of about $0.2 \mathrm{eV}$, except for the $1^{1} \mathrm{~A}_{1}$ states, whose disagreement may be attributed to involvement of the shoulder peak of this state in the intense peak at approximately $6.2 \mathrm{eV}$. For the singlet states, the lowest four excited states were assigned to $n^{+}-\pi^{+*}\left({ }^{1} \mathrm{~B}_{1}\right), n^{-}-\pi^{+*}\left({ }^{1} \mathrm{~A}_{2}\right)$, $\pi^{-}-\pi^{+*}\left({ }^{1} \mathrm{~B}_{2}\right)$, and $\pi^{+}-\pi^{+*}\left({ }^{1} \mathrm{~A}_{1}\right)$ in order of increasing energy, and the intense band at approximately $6.2 \mathrm{eV}$ in the experimental spectra was assigned to the ${ }^{1} \mathrm{~B}_{2}$ state in our calculations. For the cation doublet states, the lowest four states were assigned to $n^{+}\left({ }^{2} \mathrm{~A}_{1}\right), \pi^{-}\left({ }^{2} \mathrm{~A}_{2}\right), n^{-}\left({ }^{2} \mathrm{~B}_{2}\right)$, and $\pi^{+}\left({ }^{2} \mathrm{~B}_{1}\right)$ in order of increasing energy. Shake-up ionized states appeared in the energy region higher than $16 \mathrm{eV}$. For the anion doublet states, the ground state was ${ }^{2} \mathrm{~B}_{1}$, and five valence excited states were calculated within $4.0 \mathrm{eV}$ above the anion ground state. The adiabatic electron affinity was calculated to be $1.63 \mathrm{eV}$, which is in very good agreement with the corresponding experimental value $(1.62 \mathrm{eV})$. The use of Koopmans' theorem does not reproduce this energy order for either the singlet or the doublet states. We showed that, as in the case of $p$-BQ ( $J$. Phys. Chem. A 2002, 106, 3838), electron correlation is essential in the description of the excited states of $o$-BQ.
\end{abstract}

\section{Introduction}

Quinones are oxidized forms of aromatic diol molecules and are easily transformed to partially or fully reduced forms (e.g., the benzoquinone-hydroquinone redox system). For this reason they are frequently found as electron-acceptors in electrontransfer systems in chemistry, biology, and industrial applications. $p$-Benzoquinone ( $p$-BQ) and $o$-benzoquinone $(o$-BQ) are typical quinone compounds and, as they are the simplest reference molecules, are important in studying the electronic structures and functions of quinone species. In particular, investigation of the excited states of these compounds is necessary for understanding the nature of electron-transfer and photolysis in systems containing quinones. Although $o$-quinones are not as widespread as $p$-quinones in biological systems, they play a significant role as ligands in transition metal chemistry. Nevertheless, while the electronic states of $p$-BQ have been extensively studied both experimentally and theoretically, ${ }^{1-5}$ those of $o$-BQ have not been studied in detail because of its high reactivity. ${ }^{6,7}$

The excitation spectra of neutral $o$-BQ were experimentally observed by Goldschmidt and Graef $^{8}$ and by Meier and Wagniére. ${ }^{7}$ In order to perform assignments of the spectra, theoretical studies were conducted by several authors ${ }^{7,9-12}$ using the semiempirical methods. Kuboyama and Wada calculated singlet and triplet $\pi-\pi^{*}$ excited states of $o$-BQ using self-

\footnotetext{
* To whom correspondence should be addressed.

$\dagger$ Tokyo Metropolitan University.

$\doteqdot$ Japan Science and Technology Agency.

$\S$ Kyoto University.
}

consistent field molecular orbitals (SCF-MOs) with approximated two-electron integrals and the single excitation configuration interaction (SECI) method within a limited configuration space. ${ }^{10}$ Subsequently, Kuboyama et al. reported the singlet $\pi-\pi^{*}$ excited states of several quinones, including $o$-BQ, using the Pariser-Parr-Pople (PPP) method. ${ }^{11}$ Srivastava and Srivastava calculated the lowest five singlet $\pi-\pi^{*}$ excited states using the PPP method. ${ }^{12}$ Meier and Wagniére investigated the excited states of many quinones both experimentally and theoretically. ${ }^{7}$ These studies have established that there are at least three allowed states in the energy region below $\sim 6 \mathrm{eV}$, all of which are assigned to $\pi-\pi^{*}$ states. The lowest allowed state has been assigned to ${ }^{1} \mathrm{~B}_{2}$. However, for assignment of the second lowest $\pi-\pi^{*}$ state, Kuboyama et al. and Srivastava proposed the ${ }^{1} \mathrm{~B}_{2}$ state, whereas Meier and Wagniére proposed the ${ }^{1} \mathrm{~A}_{1}$ state; the third $\pi-\pi^{*}$ state was assigned to ${ }^{1} \mathrm{~A}_{1}$ by Kuboyama et al. and Srivastava and Srivastava but to ${ }^{1} \mathrm{~B}_{2}$ by Meier and Wagniére. Therefore, the assignment of the $\pi-\pi^{*}$ states of $o$-BQ is still unresolved. On the other hand, the existence of $n-\pi^{*}$ electronic states below the lowest $\pi-\pi^{*}$ state has been reported for neutral $p$-BQ, and therefore, it is natural to expect such $n-\pi^{*}$ states also for $o$-BQ. Meier and Wagniére observed two electronic states with very small intensities below the lowest $\pi-\pi^{*}$ state in their excitation and magnetic circular dichroism (MCD) spectra, and both were assigned to $n-\pi^{*} .7$ They also confirmed it by the PPP calculations.

There have been few studies on the ionized states of $o$-BQ. ${ }^{13,14}$ Koenig et al. ${ }^{13}$ observed the photoelectron spectrum of $o$-BQ for the lowest four states; however, these ionized states were not assigned. Schang et al. ${ }^{14}$ observed further ionized states up 
to approximately $16 \mathrm{eV}$; assignment was carried out only for the lowest four states, according to MINDO/ $3^{15}$ calculations and Koopmans' theorem, as $n, \pi, n, \pi$, in increasing energy order. This is the only theoretical study on the ionized states of $o$-BQ, and, to our knowledge, no ab initio calculations have been reported; there have also been no reports on the effects of electron correlation. Moreover, assignments for the higher ionized states have not yet been given. Thus, assignment of the ionized states of $o$-BQ is an unsolved problem.

Few studies on the electronic states of the $o-\mathrm{BQ}$ anion radical $\left(o-\mathrm{BQ}^{-}\right)$can be found, except for an experimental study by Marks et al., ${ }^{16}$ which approximately assigned the adiabatic electron affinity as $1.62 \mathrm{eV}$ on the basis of electron photodetachment spectroscopy. They also observed the dipole-bound excited states of $o-\mathrm{BQ}^{-}$below the electron-detachment threshold. However, the resonance states above the threshold have not yet been reported.

For reliable theoretical assignment of the spectra, we used the symmetry adapted cluster (SAC) ${ }^{17} / \mathrm{SAC}$-configuration interaction (SAC-CI) ${ }^{18,19}$ method, a powerful theory for describing ground and excited electronic states for various spin multiplicities which has previously been applied to several systems. ${ }^{1,20,21}$ In our previous study, we successfully employed SAC-CI to describe the electronic states of $p$-BQ and its anion radical. ${ }^{1}$ Here, we investigate the singlet excited and (cation- and anion-) doublet ground and excited states of $o$-BQ and provide assignments for the observed spectra. The calculated excitation and ionization energies show good agreement with experimental observations, which indicates that our assignments are reasonable.

\section{Computational Method}

We calculated the singlet, cation-doublet, and anion-doublet ground and excited states of $o$-BQ $\left(\mathrm{C}_{6} \mathrm{H}_{4} \mathrm{O}_{2}\right)$ by the SAC/SAC$\mathrm{CI}$ method. For calculation of the singlet states, the cc-pVTZ basis set ${ }^{22}$ plus Dunning's diffuse anion and Rydberg basis functions $^{23}$ at the center of the molecule were employed. For doublet states, we used Huzinaga-Dunning's (9s5p/4s)/[5s $3 \mathrm{p} /$ $3 \mathrm{~s}$ ] with standard scaling factors, ${ }^{24}$ Huzinaga's polarization functions $^{25}$ ([2d] for $\mathrm{C}$ and $\mathrm{O},[1 \mathrm{p}]$ for $\mathrm{H}$ ), and Dunning's diffuse anion basis ${ }^{23}$ ([1s1p] for C, [2s2p] for O with standard splitting factors); further, Dunning's Rydberg basis functions ${ }^{23}$ at the center of the molecule were added to this basis set in the aniondoublet calculations. The Rydberg basis consisted of the $3 \mathrm{~s}$, $3 p$, and $3 d$ functions of carbon, and they were split to [2s $2 p 2 d]$ with standard splitting factors.

The molecular geometry of $o$-BQ was obtained from X-ray diffraction experiments in crystal. ${ }^{26}$ For $o-\mathrm{BQ}^{-}$, the geometry was optimized by unrestricted MP2/[5s $3 \mathrm{p} / 3 \mathrm{~s}]+$ pol.(d, p) + diff.(s, p) calculations; the geometries were planar.

We used the SAC/SAC-CI SD- $R$ method, in which one- and two-electron excitation operators are considered as linked operators. Hartree-Fock (HF) orbitals were used as reference orbitals. Only 1s orbitals and their counter unoccupied orbitals were treated as frozen orbitals. All of the single excitation operators and some of the double excitation operators selected by the perturbative selection procedure ${ }^{27-29}$ were employed as linked operators. The selection thresholds were $\lambda_{\mathrm{g}}=3 \times 10^{-6}$ and $\lambda_{\mathrm{e}}=3 \times 10^{-7}$ for the SAC and SAC-CI states in the singlet calculations, $\lambda_{\mathrm{g}}=5 \times 10^{-6}$ and $\lambda_{\mathrm{e}}=5 \times 10^{-7}$ in the cationdoublet calculations, and $\lambda_{\mathrm{g}}=1 \times 10^{-6}$ and $\lambda_{\mathrm{e}}=1 \times 10^{-7}$ in the anion-doublet calculations, respectively. For the unlinked operators, $S_{2}^{2}, R_{1} S_{2}$, and $R_{2} S_{2}$ types were employed, where $S_{n}$ is a symmetry-adapted $n$-electron excitation operator for the
TABLE 1: Orbital Energies and Natures of HF Orbitals of $o-\mathrm{BQ}$

\begin{tabular}{|c|c|c|c|}
\hline MO number ${ }^{a}$ & symmetry $^{b}$ & orbital energy $[\mathrm{eV}]$ & nature $^{c}$ \\
\hline $\begin{array}{l}16 \\
17 \\
18 \\
19 \\
20 \\
21 \\
22 \\
23 \\
24 \\
25 \\
26 \\
27 \\
28\end{array}$ & $\begin{array}{l}9 \mathrm{a}_{1} \\
10 \mathrm{a}_{1} \\
8 \mathrm{~b}_{2} \\
11 \mathrm{a}_{1} \\
9 \mathrm{~b}_{2} \\
1 \mathrm{~b}_{1} \\
10 \mathrm{~b}_{2} \\
12 \mathrm{a}_{1} \\
1 \mathrm{a}_{2} \\
11 \mathrm{~b}_{2} \\
2 \mathrm{~b}_{1} \\
13 \mathrm{a}_{1} \\
2 \mathrm{a}_{2}\end{array}$ & $\begin{array}{r}\text { pied Orbitals } \\
-19.680 \\
-19.296 \\
-18.671 \\
-17.440 \\
-17.410 \\
-16.174 \\
-16.162 \\
-15.422 \\
-14.187 \\
-13.423 \\
-12.923 \\
-11.506 \\
-9.894\end{array}$ & $\begin{array}{l}\sigma^{+} \\
\sigma^{+} \\
\sigma^{-} \\
n^{+} \\
\sigma^{-} \\
\pi^{+} \\
n^{-} \\
\sigma^{+} \\
\pi^{-} \\
n^{-} \\
\pi^{+} \\
n^{+} \\
\pi^{-} \text {(HOMO) }\end{array}$ \\
\hline $\begin{array}{l}29 \\
30 \\
31 \\
32 \\
33 \\
34\end{array}$ & $\begin{array}{l}3 \mathrm{~b}_{1} \\
14 \mathrm{a}_{1} \\
12 \mathrm{~b}_{2} \\
4 \mathrm{~b}_{1} \\
15 \mathrm{a}_{1} \\
16 \mathrm{a}_{1}\end{array}$ & $\begin{array}{r}\text { upied Orbitals } \\
-0.271 \\
0.343 \\
0.614 \\
0.703 \\
0.743 \\
0.856\end{array}$ & $\begin{array}{l}\pi^{+*}(\text { LUMO }) \\
\operatorname{Ryd}\left(\sigma^{+}\right) \\
\operatorname{Ryd}\left(\sigma^{-}\right) \\
\operatorname{Ryd}\left(\pi^{+}\right) \\
\operatorname{Ryd}\left(\sigma^{+)}\right. \\
\operatorname{Ryd}\left(\sigma^{+}\right)\end{array}$ \\
\hline 39 & $18 \mathrm{a}_{1}$ & 2.165 & $\operatorname{Ryd}\left(\sigma^{+}\right)$ \\
\hline 46 & $7 b_{1}$ & 2.937 & $\pi^{+*}, \operatorname{Ryd}\left(\pi^{+}\right)$ \\
\hline 51 & $5 \mathrm{a}_{2}$ & 3.306 & $\operatorname{Ryd}\left(\pi^{-}\right)$ \\
\hline 61 & $7 \mathrm{a}_{2}$ & 4.753 & $\pi^{-*}$ \\
\hline
\end{tabular}

${ }^{a}$ Numbering starts from the lowest energy orbital including the core orbitals. ${ }^{b}$ See the text for the definition of molecular axes. ${ }^{c}$ Superscripts + and - stand for the symmetry with respect to the mirror plane between the two carbonyl groups ( $z x$ plane).

SAC calculation and $R_{n}$ is the same for the SAC-CI calculation. Selection thresholds for the unlinked terms were $\tau_{\mathrm{g}}=0.005, \tau$ $=0.0$, and $\tau_{\mathrm{s}}=\tau_{\mathrm{d}}=0.05$ in the singlet and anion-doublet calculations, and $\tau_{\mathrm{g}}=0.002, \tau=0.0008$, and $\tau_{\mathrm{s}}=\tau_{\mathrm{d}}=0.05$ in the cation-doublet calculations; the details of these thresholds are described elsewhere. ${ }^{27-29}$

All calculations were carried out using the local version of the SAC-CI program ${ }^{29}$ combined with the Gaussian 98 and Gaussian 03 program packages.

\section{Results and Discussion}

3.1. Singlet Excited States. We define the $z$ axis as the $C_{2}$ rotational axis and the $x$ axis as the direction perpendicular to the molecular plane as seen below.

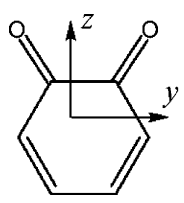

The energies and natures of the HF orbitals of neutral $o$-BQ are listed in Table 1. The MO shapes of the highest four occupied orbitals and the lowest unoccupied molecular orbitals (LUMO) are drawn in Figure 1. Superscripts + and - stand for symmetric and antisymmetric character with respect to the $z x$ plane of the molecule, respectively. The highest occupied molecular orbital (HOMO) is assigned to $\pi^{-}\left(\# 28,2 \mathrm{a}_{2}\right)$, and the next HOMO is $n^{+}\left(\# 27,13 \mathrm{a}_{1}\right)$. The lower orbitals consist of $\pi^{+}\left(\# 26,2 \mathrm{~b}_{1}\right), n^{-}\left(\# 25,11 \mathrm{~b}_{2}\right), \pi^{-}\left(\# 24,1 \mathrm{a}_{2}\right), \sigma^{+}\left(\# 23,12 \mathrm{a}_{1}\right)$, $n^{-}\left(\# 22,10 \mathrm{~b}_{2}\right), \pi^{+}\left(\# 21,1 \mathrm{~b}_{1}\right), \sigma^{-}\left(\# 20,9 \mathrm{~b}_{2}\right), n^{+}\left(\# 19,11 \mathrm{a}_{1}\right)$, and so on. For the occupied valence orbitals, four $\pi$ and four $n$ orbitals exist, and the others are assigned to $\sigma$. The LUMO (\#29, $\left.3 b_{1}\right)$ is a $\pi^{+*}$ orbital, which is to be the singly occupied 


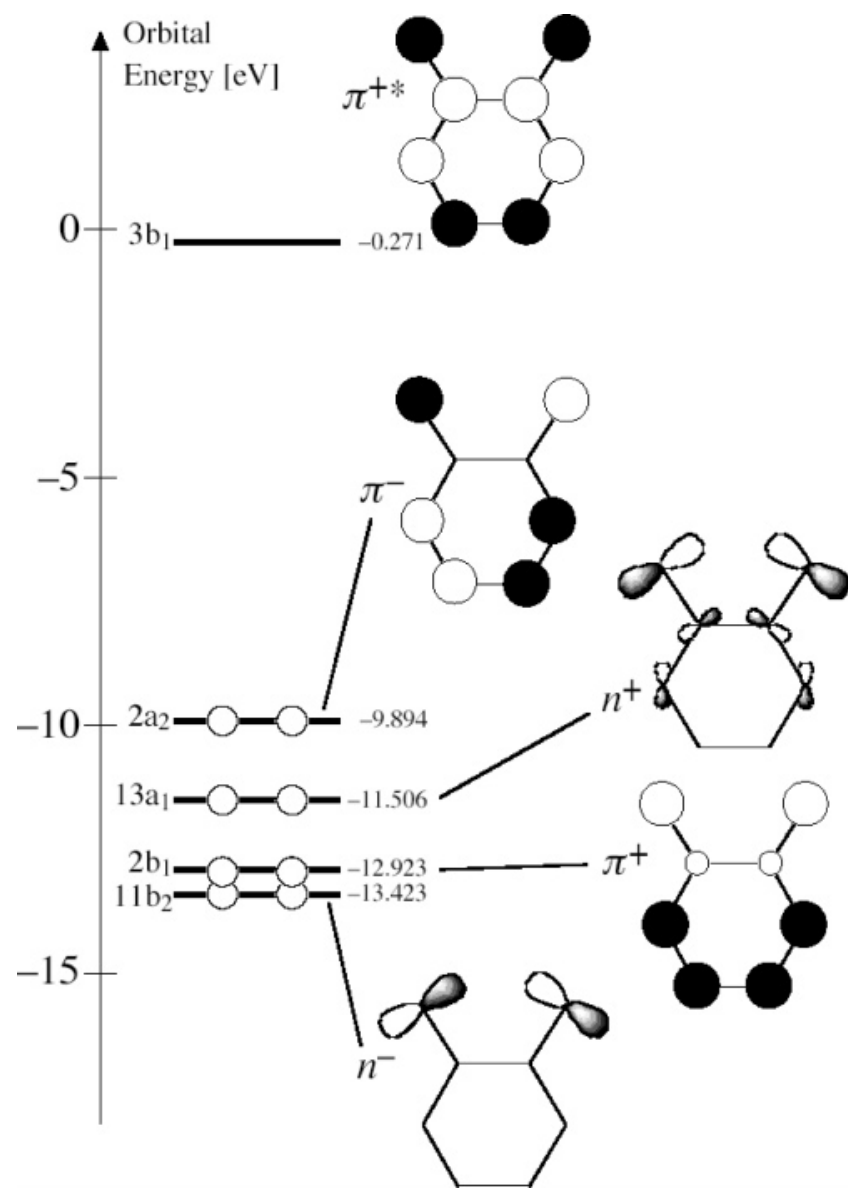

Figure 1. Some important HF MOs and their energies of $o$-BQ. Superscripts + and - stand for the symmetry for the mirror plane between the two carbonyl groups ( $z x$ plane).

molecular orbital (SOMO) for the $o$-BQ anion radical. The orbital energy of the LUMO is negative $(-0.271 \mathrm{eV})$, suggesting that the electron affinity of $o$-BQ is largely positive. The experimental results, ${ }^{16}$ along with our SAC-CI results, support this supposition, as seen later. Two $\pi^{*}$ orbitals (\#46, $7 b_{1}$ and $\# 61,7 a_{2}$ ) exist in the unoccupied higher energy region. There are many Rydberg orbitals among these $\pi^{*}$ orbitals.
The excitation energies, oscillator strengths, main configurations, and natures of the singlet states as calculated by SAC-CI are shown in Table 2, which also shows the experimental results. ${ }^{7,8}$ The calculated values show good agreement with the experimental results, although the excitation energy of the $1^{1} \mathrm{~B}_{1}$ state was somewhat underestimated. Apart from that of the $1{ }^{1} \mathrm{~A}_{1}$ state, the mean error in the theoretical and experimental excitation energies was about $0.2 \mathrm{eV}$. The overestimation of the excitation energy of the $1{ }^{1} \mathrm{~A}_{1}$ state may be attributed to involvement of the shoulder peak of the $1{ }^{1} \mathrm{~A}_{1}$ state in the intense peak of the $2{ }^{1} \mathrm{~B}_{2}$ state.

Table 3 shows a comparison of the present SAC-CI results with those previously reported. , $8,10-12^{2}$ The lowest and second lowest excited states, according to our calculations, were the $n^{+}-\pi^{+*}\left({ }^{1} \mathrm{~B}_{1}\right)$ and $n^{-}-\pi^{+*}\left({ }^{1} \mathrm{~A}_{2}\right)$ states, respectively. Calculations by Meier and Wagniére ${ }^{7}$ support our results. The extra peak at approximately $2.1 \mathrm{eV}$ in the experimental spectrum in ref 7 is thought to be due to vibrational excitation. The third state was assigned as $\pi^{-}-\pi^{+*}\left({ }^{1} \mathrm{~B}_{2}\right)$. This result is in accordance with other reported assignments. In our calculations, the fourth excited state was calculated to be the $\pi^{+}-\pi^{+*}\left({ }^{1} \mathrm{~A}_{1}\right)$ state, and the intense band at approximately $6.2 \mathrm{eV}$ in the experimental spectrum was assigned to $\pi^{-}-\pi^{+*}\left({ }^{1} \mathrm{~B}_{2}\right)$. These results agree with the assignments of Meier and Wagniére ${ }^{7}$ and differ from those of Kuboyama et al. ${ }^{10,11}$ and Srivastava and Srivastava, ${ }^{12}$ as

Table 3. The calculated oscillator strengths of these three $\pi-\pi^{*}$ states were $0.0711,0.0759$, and 0.1600 , in order of increasing energy; these trends are in good agreement with the experimental spectral shapes. Similarly to the reported theoretical results, ${ }^{10-12}$ our calculations predict two $\pi-\pi^{*}$ excited states above the highest bands observed experimentally (ca. $6.2 \mathrm{eV}$ ); these were calculated as 6.84 and $7.27 \mathrm{eV}$ and are assigned to $\pi^{-}-\pi^{+*}$ $\left({ }^{1} \mathrm{~B}_{2}\right)$ and $\pi^{-}-\pi^{-*}\left({ }^{1} \mathrm{~A}_{1}\right)$, respectively. Besides these valence excited states, many Rydberg excited states were also calculated. Almost all of them are associated with excitation from the highest four occupied orbitals, and the assignments are given in Table 2.

A comparison of the order of the lowest four excited states as calculated by SAC-CI (Table 2) to the HF-calculated states (Table 1) reveals changes in the energy order; namely, SACCI gives $n^{+-}-\pi^{+*}\left({ }^{1} \mathrm{~B}_{1}\right), n^{-}-\pi^{+*}\left({ }^{1} \mathrm{~A}_{2}\right), \pi^{-}-\pi^{+*}\left({ }^{1} \mathrm{~B}_{2}\right)$, and $\pi^{+-}$

TABLE 2: Excitation Energies, Oscillator Strengths, Main Configurations, and Natures of the Singlet Excited States of $o$-BQ

\begin{tabular}{|c|c|c|c|c|c|}
\hline \multicolumn{4}{|c|}{ SAC-CI } & \multicolumn{2}{|c|}{ exptl } \\
\hline states & coefficients of main configurations $(|C|>0.3)$ & nature & $\begin{array}{c}\text { excitation } \\
\text { energy }^{a}[\mathrm{eV}]\end{array}$ & $\begin{array}{c}\text { Meier and } \\
\text { Wagniere }^{a, b}[\mathrm{eV}]\end{array}$ & $\begin{array}{c}\text { Goldschmidt } \\
\text { and Graef }{ }^{a, c}[\mathrm{eV}]\end{array}$ \\
\hline $1{ }^{1} \mathrm{~B}_{1}$ & $0.91(27-29)$ & $n^{+}-\pi^{+*}$ LUMO & $1.63(0.0000)$ & 2.0 (very small) & \\
\hline $1{ }^{1} \mathrm{~A}_{2}$ & $0.87(25-29)$ & $n^{-}-\pi^{+*}$ LUMO & $2.95(0.0000)$ & 2.7 (very small) & \\
\hline $1{ }^{1} \mathrm{~B}_{2}$ & $0.95(28-29)$ & $\pi^{-}-\pi^{+*}{ }_{\text {LUMO }}$ & $3.24(0.0732)$ & $<3.2(0.039)$ & $3.5(0.05)$ \\
\hline $1{ }^{1} \mathrm{~A}_{1}$ & $0.79(26-29)$ & $\pi^{+}-\pi^{+*}$ LUMO & $5.67(0.0778)$ & $<4.8(\sim 0.025)$ & $4.9(\sim 0.03)$ \\
\hline $2{ }^{1} \mathrm{~B}_{1}$ & $0.64(27-46)-0.38(27-62)+0.32(27-59)$ & $n^{+}-\pi^{+*}, \operatorname{Ryd}\left(\pi^{+}\right)$ & $5.76(0.0000)$ & & \\
\hline $2{ }^{1} \mathrm{~A}_{2}$ & $0.54(27-51)+0.49(27-61)+0.43(27-57)$ & $n^{+}-\pi^{-*}, \operatorname{Ryd}\left(\pi^{-}\right)$ & $6.22(0.0000)$ & & \\
\hline $3{ }^{1} \mathrm{~A}_{2}$ & $0.82(28-30)-0.43(28-39)$ & $\pi^{-}-\operatorname{Ryd}\left(\sigma^{+}\right)$ & $6.39(0.0000)$ & & \\
\hline $2{ }^{1} \mathrm{~A}_{1}$ & $0.77(27-30)-0.39(27-39)+0.32(27-33)$ & $n^{+}-\operatorname{Ryd}\left(\sigma^{+}\right)$ & $6.45(0.0001)$ & & \\
\hline $2{ }^{1} \mathrm{~B}_{2}$ & $0.75(24-29)-0.38(28-46)$ & $\pi^{-}-\pi^{+*}$ LUMO & $6.46(0.1640)$ & $\sim 6.2$ (very large) & $\sim 6.2(>0.2)$ \\
\hline $3{ }^{1} \mathrm{~B}_{1}$ & $0.88(23-29)$ & $\sigma^{+}-\pi^{+*}$ LUMO & $6.61(0.0003)$ & & \\
\hline $3{ }^{1} \mathrm{~A}_{2}$ & $0.86(22-29)$ & $n^{-}-\pi^{+*}$ LUMO & $6.68(0.0000)$ & & \\
\hline $3{ }^{1} \mathrm{~B}_{2}$ & $0.62(28-46)+0.48(24-29)$ & $\pi^{-}-\pi^{+*}$ & $6.84(0.0259)$ & & \\
\hline $3{ }^{1} \mathrm{~A}_{1}$ & $0.48(27-30)-0.43(27-33)-0.42(27-38)+0.31(27-44)$ & $n^{+}-\operatorname{Ryd}\left(\sigma^{+}\right)$ & $7.00(0.0019)$ & & \\
\hline $4{ }^{1} \mathrm{~B}_{2}$ & $0.78(27-31)-0.42(27-36)$ & $n^{+}-\operatorname{Ryd}\left(\sigma^{-}\right)$ & $7.15(0.0298)$ & & \\
\hline $4{ }^{1} \mathrm{~B}_{1}$ & $0.85(28-31)$ & $\pi^{-}-\operatorname{Ryd}\left(\sigma^{-}\right)$ & $7.18(0.0182)$ & & \\
\hline $4{ }^{1} \mathrm{~A}_{2}$ & $0.59(28-33)-0.35(28-44)+0.33(28-34)-0.32(28-39)$ & $\pi^{-}-\operatorname{Ryd}\left(\sigma^{+}\right)$ & $7.19(0.0000)$ & & \\
\hline $5{ }^{1} \mathrm{~B}_{2}$ & $0.76(28-32)-0.40(28-43)$ & $\pi^{-}-\operatorname{Ryd}\left(\pi^{+}\right)$ & $7.25(0.0000)$ & & \\
\hline $4{ }^{1} \mathrm{~A}_{1}$ & $0.48(28-51)+0.40(26-29)+0.38(28-61)+0.35(28-57)$ & $\pi^{-}-\pi^{-*}, \operatorname{Ryd}\left(\pi^{-}\right)$ & $7.27(0.7591)$ & & \\
\hline $5{ }^{1} \mathrm{~A}_{1}$ & $0.67(27-34)+0.29(27-33)$ & $n^{+}-\operatorname{Ryd}\left(\sigma^{+}\right)$ & $7.65(0.0431)$ & & \\
\hline
\end{tabular}

\footnotetext{
${ }^{a}$ Values in parentheses are the oscillator strengths. ${ }^{b}$ Reference $7 .{ }^{c}$ Reference 8.
} 
TABLE 3: Comparison of the Calculated and Observed Excitation Energies of $o-B^{a}{ }^{a}$

\begin{tabular}{|c|c|c|c|c|c|c|}
\hline \multicolumn{5}{|c|}{ theoretical } & \multicolumn{2}{|c|}{ experimental } \\
\hline $\begin{array}{c}\text { SAC-CI } \\
\text { (present results) }\end{array}$ & $\begin{array}{c}\text { CNDO/S } \\
\text { Meier and Wagniere }{ }^{b}\end{array}$ & $\begin{array}{l}\text { PPP, Srivastava } \\
\text { and Srivastava }{ }^{c}\end{array}$ & $\begin{array}{c}\text { PPP } \\
\text { Kuboyama et al. }^{d}\end{array}$ & $\begin{array}{l}\text { Approximately SECI } \\
\text { Kuboyama and Wada }{ }^{e}\end{array}$ & $\begin{array}{l}\text { Meier and } \\
\text { Wagniere }^{b}\end{array}$ & $\begin{array}{l}\text { Goldschmidt } \\
\text { and Graeff }\end{array}$ \\
\hline $1.63(0.0000) /{ }^{1} \mathrm{~B}_{1}$ & $1.96(0.0) /{ }^{1} \mathrm{~B}_{1}$ & & & & 2.0 (small) & \\
\hline $2.95(0.0000) /{ }^{1} \mathrm{~A}_{2}$ & $2.96(0.0) /{ }^{1} \mathrm{~A}_{2}$ & & & & 2.7 (small) & \\
\hline $3.24(0.0732) /{ }^{1} \mathrm{~B}_{2}$ & $\sim 3.4(\sim 0.03) /{ }^{1} \mathrm{~B}_{2}$ & $3.17(0.28) /{ }^{1} \mathrm{~B}_{2}$ & $3.72(0.234) /{ }^{1} \mathrm{~B}_{2}$ & $5.17 /{ }^{1} \mathrm{~B}_{2}$ & $<3.2(0.039)$ & $3.5(0.05)$ \\
\hline $5.67(0.0778) /{ }^{1} \mathrm{~A}_{1}$ & $\sim 5.4(\sim 0.03) /{ }^{1} \mathrm{~A}_{1}$ & $4.66(0.06) /{ }^{1} \mathrm{~B}_{2}$ & $5.44(0.146) /{ }^{1} \mathrm{~B}_{2}$ & $6.61 /{ }^{1} \mathrm{~B}_{2}$ & $<4.8(\sim 0.025)$ & $4.9(\sim 0.03)$ \\
\hline $6.46(0.1640) /{ }^{1} \mathrm{~B}_{2}$ & $\sim 6.1(0.14) /{ }^{1} \mathrm{~B}_{2}$ & $4.93(0.14) /{ }^{1} \mathrm{~A}_{1}$ & $6.31(0.146) /{ }^{1} \mathrm{~A}_{1}$ & $6.95 /{ }^{1} \mathrm{~A}_{1}$ & $\sim 6.2$ (large) & $\sim 6.2(>0.2)$ \\
\hline $6.84(0.0259) /{ }^{1} \mathrm{~B}_{2}$ & & $5.41(0.26) /{ }^{1} \mathrm{~B}_{2}$ & $7.42(0.405) /{ }^{1} \mathrm{~A}_{1}$ & $7.29 /{ }^{1} \mathrm{~B}_{2}$ & & \\
\hline $7.27(0.7591) /{ }^{1} \mathrm{~A}_{1}$ & & $6.07(0.93) /{ }^{1} \mathrm{~A}_{1}$ & $7.64(0.216) /{ }^{1} \mathrm{~B}_{2}$ & $7.50 /{ }^{1} \mathrm{~A}_{1}$ & & \\
\hline
\end{tabular}

${ }^{a}$ Each cell means "excitation energy [eV] (oscillator strength)/assignment". ${ }^{b}$ Reference $7 .{ }^{c}$ Reference 12. ${ }^{d}$ Reference $11 .{ }^{e}$ Reference 10. ${ }^{f}$ Reference 8 .



Figure 2. Singlet excitation energies of the four lowest excited states for six calculations; $p$-BQ/o-BQ obtained by HF/SECI/SAC-CI. The circles and solid lines stand for $\pi$-ionized states, while the triangles and dashed lines represent $n$-ionized states. The excitation energies for $p$-BQ are taken from ref 1 . See the text for the meaning of the superscripts for $o-\mathrm{BQ}$, and the subscripts and superscripts for $p$-BQ stand for the symmetries with respect to the inversion center and the mirror plane intersecting the two $\mathrm{C}=\mathrm{C}$ bonds in $p-\mathrm{BQ}$, respectively. $\pi^{+*}\left({ }^{1} \mathrm{~A}_{1}\right)$ as the lowest four excited states in order of increasing energy, while HF gives $\pi^{-}-\pi^{+*}\left({ }^{1} \mathrm{~B}_{2}\right), n^{+}-\pi^{+*}\left({ }^{1} \mathrm{~B}_{1}\right), \pi^{+-}$ $\pi^{+*}\left({ }^{1} \mathrm{~A}_{1}\right)$, and $n^{-}-\pi^{+*}\left({ }^{1} \mathrm{~A}_{2}\right)$.

This is attributed to orbital relaxation rather than electron correlation. Figure 2 shows the excitation energies of the lowest four excited states obtained by six calculations: $p$-BQ calculated by $\mathrm{HF}$, single excitation $\mathrm{CI}$ (SECI), and SAC-CI, and $o-\mathrm{BQ}$ calculated by HF, SECI, and SAC-CI. The HF excitation energies are estimated on the basis of energy differences between the orbitals that participate in the main configurations (for example, the energy difference between the 27th and the 29th MOs in the case of $\left.1{ }^{1} \mathrm{~B}_{1}\right)$. The excitation energies for $p$-BQ are taken from our previous study. ${ }^{1}$ The energy shift between the HF and the SECI calculations in Figure 2 corresponds approximately to orbital relaxation, and that between the SECI and the SAC-CI calculations corresponds to electron correlation. For $o-\mathrm{BQ}$, the orbital relaxation effect reduces the excitation energies of the $n-\pi^{*}$ transitions more than those of the $\pi-\pi^{*}$ transitions, resulting in a change of order between the two pairs of $n-\pi^{*}$ and $\pi-\pi^{*}$ states. Furthermore, electron correlation also encourages this trend and causes order reversal of the $n^{-}-\pi^{+*}\left({ }^{1} \mathrm{~A}_{2}\right)$ and $\pi^{-}-\pi^{+*}\left({ }^{1} \mathrm{~B}_{2}\right)$ states. The situation for $p$-BQ is similar to that of $o-\mathrm{BQ}$. This is a characteristic common to $\pi$-conjugate molecules with lone pairs which do not participate in the conjugate system, and can be explained in terms of the local character of the electrons in the $n$ orbitals as compared with the $\pi$-orbitals.

3.2. Ionized Ground and Excited States. The SAC-CI results for the ionized states are shown in Table 4, which also shows the experimental ${ }^{13,14}$ and MINDO/3 calculated values. ${ }^{14}$ The intensities of the ionization bands were calculated by monopole approximation. ${ }^{30}$ Figure 3 shows the theoretical ionization spectrum obtained by SAC/SAC-CI calculations together with the experimental spectra reported by Schang et al. ${ }^{14}$ Since the calculated spectrum shows good agreement with the experimental one, we were able to assign the ionization bands listed in Table 4 with reliable accuracy. Shake-up ionized states, whose main configurations arise from one-electron excitation and one-electron ionization processes, appear in the energy region higher than $16 \mathrm{eV}$. The contribution of these processes to the spectrum is small. The shake-up processes are mainly due to $\pi-\pi^{*}$ LUMO $+n$ ionization or $n-\pi^{*}$ LUMO $+\pi$ ionization.

For $o$-BQ, Koopmans' theorem gives incorrect ordering of the ionization bands. The order of the ionized states calculated by the SAC-CI method is $n^{+}\left({ }^{2} \mathrm{~A}_{1}\right), \pi^{-}\left({ }^{2} \mathrm{~A}_{2}\right), n^{-}\left({ }^{2} \mathrm{~B}_{2}\right), \pi^{+}\left({ }^{2} \mathrm{~B}_{1}\right)$, in order of increasing energy (Table 4), while Koopmans' theorem gives $\pi^{-}\left(\mathrm{a}_{2}\right), n^{+}\left(\mathrm{a}_{1}\right), \pi^{+}\left(\mathrm{b}_{1}\right), n^{-}\left(\mathrm{b}_{2}\right)$ (Table 1$)$. The SAC-CI assignments agree with those of MINDO/ $3^{14}$ for the lowest four ionized states. The energy reversal of the $n$ and $\pi$ states as calculated by the HF and SAC/SAC-CI methods is attributed to electron correlation, as for $p$-BQ. Figure 4 shows the ionization energies of the lowest four ionized states obtained by four calculations: $p$-BQ calculated by HF and SAC-CI and $o$-BQ calculated by $\mathrm{HF}$ and SAC-CI. The ionization energies for $p$-BQ are taken from our previous study. ${ }^{1}$ In the case of both $p$-BQ and $o-\mathrm{BQ}$, electron correlation reduces the ionization energies of the $n$-ionized states by approximately $2.5 \mathrm{eV}$, while the energies of the $\pi$-ionized states are not significantly affected. This trend is also found in many $\pi$-conjugate molecules containing lone pairs. Similar to the case of singlet excitation, this can be explained by the local character of the electrons in the $n$ orbitals compared to the $\pi$ orbitals. The difference in the energy order of the ionized states of $o$-BQ $(n, \pi, n, \pi)$ and those of $p$-BQ $(n, n, \pi, \pi)$ is thus attributed to the order of the HF orbital energies.

The ionization bands (and shoulders) in the energy region greater than $13 \mathrm{eV}$ in the observed spectra correspond one-byone to the calculated states, and these were assigned to $\pi^{-}, \sigma^{+}$, $n^{-}, \pi^{+}$, and $n^{+}$states in order of increasing energy. According to our assignment, the experimental bands at $15 \mathrm{eV}$ consist of two ionization bands, $\pi^{+}$and $n^{+}$. Although no experimental data are available, we also calculated the ionized states above $15 \mathrm{eV}$ and assigned them to $\sigma^{-}, \sigma^{+}, \sigma^{-}, \sigma^{-}, \sigma^{+}$states in order 
TABLE 4: Ionization Energies and Natures of $o$-BQ

\begin{tabular}{|c|c|c|c|c|c|c|}
\hline \multicolumn{5}{|c|}{ SAC-CI } & \multirow{2}{*}{ 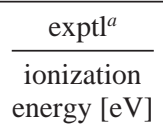 } & \multirow{2}{*}{$\begin{array}{c}\mathrm{MINDO} / 3^{a} \\
\text { ionization } \\
\text { energy }[\mathrm{eV}]\end{array}$} \\
\hline states $^{b}$ & nature $^{b}$ & $\begin{array}{c}\text { coefficients of main } \\
\text { configurations }(|C|>0.3)\end{array}$ & $\begin{array}{l}\text { ionization } \\
\text { energy }[\mathrm{eV}]\end{array}$ & intensity $^{c}$ & & \\
\hline $1{ }^{2} \mathrm{~A}_{1}$ & $n^{+}$ & $0.95(27)$ & 9.32 & 0.913 & $9.60,9.6^{d}$ & 9.18 \\
\hline $1{ }^{2} \mathrm{~A}_{2}$ & $\pi^{-}$ & $0.97(28)$ & 9.85 & 0.947 & $9.98,9.99^{d}$ & 9.84 \\
\hline $1^{2} \mathrm{~B}_{2}$ & $n^{-}$ & $0.93(25)$ & 10.71 & 0.880 & $10.88,11.0^{d}$ & 10.84 \\
\hline $1^{2} \mathrm{~B}_{1}$ & $\pi^{+}$ & $0.95(26)$ & 12.39 & 0.907 & $12.16,12.3^{d}$ & 12.25 \\
\hline $2^{2} \mathrm{~A}_{2}$ & $\pi^{-}$ & $0.94(24)$ & 13.32 & 0.894 & $13.0^{e}$ & \\
\hline $2^{2} \mathrm{~A}_{1}$ & $\sigma^{+}$ & $0.94(23)$ & 13.99 & 0.912 & $13.7^{e}$ & \\
\hline $2^{2} \mathrm{~B}_{2}$ & $n^{-}$ & $0.93(21)$ & 14.33 & 0.906 & $14.1^{e}$ & \\
\hline $2^{2} B_{1}$ & $\pi^{+}$ & $0.89(22)-0.33(28-29,24)$ & 14.95 & 0.794 & $(15.0)^{e, f}$ & \\
\hline $3{ }^{2} \mathrm{~A}_{1}$ & $n^{+}$ & $0.93(20)$ & 15.10 & 0.891 & $15.1^{e}$ & \\
\hline $3{ }^{2} \mathrm{~B}_{2}$ & $\sigma^{-}$ & $0.82(19)-0.39(27-29,28)$ & 15.95 & 0.778 & & \\
\hline $4{ }^{2} \mathrm{~A}_{1}$ & $\sigma^{+}$ & $0.83(17)-0.39(28-29,25)$ & 16.81 & 0.723 & & \\
\hline $4{ }^{2} \mathrm{~B}_{2}$ & $\sigma^{-},\left(\pi^{-}-\pi^{+*}+n^{+}\right)$ & $0.61(18)+0.55(28-29,27)$ & 16.81 & 0.421 & & \\
\hline $5^{2} \mathrm{~B}_{2}$ & $\sigma^{-},\left(\pi^{-}-\pi^{+*}+n^{+}\right)$ & $0.65(18)-0.44(28-29,27)$ & 17.02 & 0.493 & & \\
\hline $5^{2} \mathrm{~A}_{1}$ & $\sigma^{+}$ & $0.92(16)$ & 18.19 & 0.868 & & \\
\hline
\end{tabular}

${ }^{a}$ Reference $14 .{ }^{b}$ Superscripts + and - stand for the symmetry with respect to the mirror plane between the two carbonyl groups ( $z x$ plane). ${ }^{c}$ Estimated using the monopole approximation. ${ }^{d}$ Reference $13 .{ }^{e}$ Values in italic are not given by the original authors. ${ }^{f}$ Value in parentheses is the energy of the shoulder band.



Figure 3. SAC-CI ionization spectrum compared with the experimental spectra observed by Schang et al. ${ }^{14}$

of increasing energy. These will form three ionization bands, as seen in Figure 3.

3.3. Anion Ground and Excited States. We employed the restricted open-shell Hartree-Fock (ROHF)-MOs of $o-\mathrm{BQ}^{-}$ as reference orbitals in the calculations of the anion-doublet ground and excited states. In this case, the ground state is represented as a SAC-CI state, not as a SAC state. For details of the ROHF-reference SAC-CI method, see ref 1.

In $o-\mathrm{BQ}^{-}$, one extra electron is accommodated in $\pi^{+*}(\# 29$, $3 b_{1}$ ), which makes this a SOMO. The ground state of the anion radical is therefore ${ }^{2} \mathrm{~B}_{1}$. The optimized geometry for $o-\mathrm{BQ}^{-}$



Figure 4. Ionization energies of the four lowest ionized states for four calculations; $p$-BQ/o-BQ obtained by HF/SAC-CI. The circles and solid lines stand for $\pi$-ionized states, while the triangles and dashed lines represent $n$-ionized states. The ionization energies for $p$-BQ are taken from ref 1 . See the text for the meaning of the superscripts for $o-\mathrm{BQ}$, and the subscripts and superscripts for $p-\mathrm{BQ}$ stand for the symmetries with respect to the inversion center and the mirror plane intersecting the two $\mathrm{C}=\mathrm{C}$ bonds in $p$-BQ, respectively.

showed longer $\mathrm{C}=\mathrm{O}$ and $\mathrm{C}=\mathrm{C}$ bonds and shorter $\mathrm{C}-\mathrm{C}$ bonds than the experimentally obtained neutral geometry. The $\mathrm{C}=\mathrm{O}$, $\mathrm{C}=\mathrm{C}$, and $\mathrm{C}-\mathrm{C}$ bond lengths in the neutral molecule were reported to be $1.220,1.341$, and $1.454 \sim 1.552 \AA$, respectively, ${ }^{26}$ while for the anion they were calculated to be 1.2504, 1.3673, and $1.3673 \sim 1.5052 \AA$, respectively. These geometric variations can be simply explained in terms of the shape of the SOMO of the anion, which shows an antibonding nature for the $\mathrm{C}=\mathrm{C}$ and $\mathrm{C}=\mathrm{O}$ bonds and a bonding nature for the $\mathrm{C}-\mathrm{C}$ bonds. Electron accommodation in the SOMO causes decreases in the $\mathrm{C}=\mathrm{C}$ and $\mathrm{C}=\mathrm{O}$ bond orders and an increase in the $\mathrm{C}-\mathrm{C}$ bond order, resulting in variations in geometry. Thus, electron attachment promotes aromaticity in $o$-BQ, and the situation is similar to that of $p$-BQ.

The excitation energies and natures of the energy states of $o-\mathrm{BQ}^{-}$, calculated by SAC-CI, are shown in Table 5. All the excitation energies in Table 5 are from the anion ground state $\left({ }^{2} \mathrm{~B}_{1}\right)$. The adiabatic electron affinity was calculated to be 1.63 $\mathrm{eV}$, which is in very good agreement with the corresponding 
TABLE 5: Energies and Natures of Excited States for $o-B^{-}$

\begin{tabular}{|c|c|c|c|c|}
\hline \multicolumn{4}{|c|}{ SAC-CI } & \multirow{2}{*}{$\frac{\text { exptl }}{{\text { Marks et al. }[\mathrm{eV}]^{a}}^{a}}$} \\
\hline states & coefficients of main configurations $(|C|>0.3)$ & nature & excitation energy [eV] (osc. str.) & \\
\hline $1{ }^{2} \mathrm{~B}_{1}$ & (anion ground state) & & adiabatic $\mathrm{EA}=1.63$ & adiabatic $\mathrm{EA}=1.62$ \\
\hline $1{ }^{2} \mathrm{~A}_{1}$ & $0.96(27-29)$ & $n^{+}-\pi^{+*}$ sOMO & $2.11(0.0000)$ & \\
\hline $1{ }^{2} \mathrm{~A}_{2}$ & $0.95(28-29)$ & $\pi^{-}-\pi^{+*}$ момо & $2.61(0.0399)$ & \\
\hline $1{ }^{2} \mathrm{~B}_{2}$ & $0.95(26-29)$ & $n^{-}-\pi^{+*}$ SOMO & $3.16(0.0000)$ & \\
\hline $2{ }^{2} \mathrm{~B}_{1}$ & $0.51(29-50)-0.49(29-65)+0.44(29-69)-0.35(29-55)$ & $\pi^{+*} \mathrm{SOMO}-\pi^{+*}$ & $3.54(0.1040)$ & \\
\hline $2{ }^{2} \mathrm{~A}_{2}$ & $0.67(29-68)-0.50(29-52)-0.36(29-64)$ & $\pi^{+*} \mathrm{sOMO}-\pi^{-*}$ & $3.95(0.0977)$ & \\
\hline
\end{tabular}

experimental value, $1.62 \mathrm{eV} .{ }^{16}$ Five valence excited states were calculated below $4.0 \mathrm{eV}$, although no experimental data have been reported for the anion radical. These states have higher energies than the neutral ground state and would be observed as resonance states. The $1{ }^{2} \mathrm{~A}_{1}, 1{ }^{2} \mathrm{~A}_{2}$, and $1{ }^{2} \mathrm{~B}_{2}$ states are formed by doubly occupied MO $\rightarrow$ SOMO excitations; these are Feshbach resonance states. The $2{ }^{2} \mathrm{~B}_{1}$ and $2{ }^{2} \mathrm{~A}_{2}$ states are due to $\mathrm{SOMO} \rightarrow$ higher unoccupied MO excitation; these are shape resonance states. The calculated second moments of these states were as large as those of the anion ground state. Therefore, the states shown in Table 5 are expected to be "real", not artificial, states. The lowest symmetry allowed excited state is assigned to $\pi^{-}-\pi^{+*}$ SOMO $\left(1^{2} \mathrm{~A}_{2}\right)$, and the second and third allowed states are assigned to $\pi^{+*}$ somo $-\pi^{+*}\left(2{ }^{2} \mathrm{~B}_{1}\right)$ and $\pi^{+*}$ sOMO $-\pi^{-*}(2$ ${ }^{2} A_{2}$ ), respectively. This order of states is different from that of $p$-BQ. According to our previous calculations for $p$-BQ, allowed excited states were assigned to $\pi_{\mathrm{g}}{ }^{-*} \mathrm{SOMO}-\pi_{\mathrm{u}}{ }^{-*}\left(1^{2} \mathrm{~A}_{\mathrm{u}}\right), \pi_{\mathrm{u}}{ }^{+-}$ $\pi_{\mathrm{g}}{ }^{-*}$ SOMO $\left(2{ }^{2} \mathrm{~B}_{3 \mathrm{u}}\right)$, and $\pi_{\mathrm{g}}{ }^{-*}$ SOMO $-\pi_{\mathrm{u}}{ }^{+}\left({ }^{2} \mathrm{~B}_{3 \mathrm{u}}\right)$ (the subscripts and superscripts in the natures represent the symmetries with respect to the inversion center and the mirror plane intersecting the two $\mathrm{C}=\mathrm{C}$ bonds in $p$-BQ, respectively) in order of increasing energy. ${ }^{1}$ This difference is accounted for in terms of the energy difference between the $\pi_{\mathrm{u}}{ }^{+}$orbital of $p$-BQ and the $\pi^{-}$orbital of $o-\mathrm{BQ}$, as seen in Figures 2 and 4. Besides these valence states, many excited states with diffuse characters were calculated in this energy region. However, at this stage we do not know whether these states are real or artificial, and calculations with enlarged diffuse bases would be required to obtain conclusive results.

\section{Summary}

Singlet excited and ionized states of $o-\mathrm{BQ}$ and doublet ground and excited states of the $o$-BQ anion radical $\left(o-\mathrm{BQ}^{-}\right)$were studied by the SAC/SAC-CI method, and the states were assigned. The SAC-CI theoretical spectra were in good agreement with the experimental results, except for the $1^{1} \mathrm{~B}_{1}$ and $1^{1} \mathrm{~A}_{1}$ states. (The overestimation of the excitation energy of the $1^{1} \mathrm{~A}_{1}$ state may be attributed to involvement of the shoulder peak of the $1{ }^{1} \mathrm{~A}_{1}$ state in the intense peak of the $2{ }^{1} \mathrm{~B}_{2}$ state.) The lowest four excited states were calculated as $n^{+}-\pi^{+*}\left({ }^{1} \mathrm{~B}_{1}\right)$, $n^{-}-\pi^{+*}\left({ }^{1} \mathrm{~A}_{2}\right), \pi^{-}-\pi^{+*}\left({ }^{1} \mathrm{~B}_{2}\right)$, and $\pi^{+}-\pi^{+*}\left({ }^{1} \mathrm{~A}_{1}\right)$, in order of increasing energy. The intense band at approximately $6.2 \mathrm{eV}$ in the experimental spectrum was assigned to the ${ }^{1} \mathrm{~B}_{2} \pi^{-}-\pi^{+*}$ state, in agreement with the assignment by Meier and Wagniére. The calculated ionized states were assigned to $n^{+}, \pi^{-}, n^{-}, \pi^{+}$, $\pi^{-}, \sigma^{+}, n^{-}, \pi^{+}, n^{+}, \sigma^{-}, \sigma^{+}, \sigma^{-}, \sigma^{-}, \sigma^{+}$in order of increasing energy. Shake-up ionized states appeared in the energy region higher than $16 \mathrm{eV}$. For the anion-doublet calculations, the ground state was ${ }^{2} \mathrm{~B}_{1}$, and the adiabatic electron affinity was calculated to be $1.63 \mathrm{eV}$, which is in very good agreement with the corresponding experimental result $(1.62 \mathrm{eV})$. The excited states of $o-\mathrm{BQ}^{-}$were assigned to $n^{+}-\pi^{+*}$ somo, $\pi^{-}-\pi^{+*}$ somo, $n^{-}-\pi^{+*}$ sOMO, $\pi^{+*}$ SOMO $^{-} \pi^{+*}$, and $\pi^{+*}$ SOMO $-\pi^{-*}$, in order of increasing energy. These SAC-CI results are different from Koopmans' energy order. Thus, it is essential to take electron correlation into account when describing the singlet and doublet excited states of $o-\mathrm{BQ}$. This is similar to the case of $p-\mathrm{BQ}$.

Acknowledgment. Part of this study has been supported by a Grant-in-Aid for Scientific Research from the Ministry of Education, Culture, Sports, Science, and Technology of Japan.

\section{References and Notes}

(1) Honda, Y.; Hada, M.; Ehara, M.; H. Nakatsuji, J. Phys. Chem. A 2002, 106, 3838. Chief experimental and theoretical studies on the ground and excited states of neutral, ionized, and anionized $p$-benzoquinone are referred to therein.

(2) Schiedt, J.; Weinkauf, R. J. Chem. Phys. 1999, 110, 304. 9536.

(3) Pou-Amérigo, R.; Merchán, M.; Ortí, E. J. Chem. Phys. 1999, 110,

(4) Pou-Amérigo, R.; Serrano-Andrés, L.; Merchán, M.; Ortí, E.; Forsberg, N. J. Am. Chem. Soc. 2000, 122, 6067.

(5) Stanton, J. F.; Sattelmeyer, K. W.; Gauss, J.; Allan, M.; Skalicky, T.; Bally, T. J. Chem. Phys. 2001, 115, 1.

(6) Tomioka, H.; Fukao, H.; Izawa, Y. Bull. Chem. Soc. Jpn. 1978, 51,540 .

(7) Meier, A. R.; Wagniére, G. H. Chem. Phys. 1987, 113, 287.

(8) Goldschmidt, S.; Graef, F. Ber. Dtsch. Chem. Ges. 1928, 61, 1858.

(9) Bery, J. C. Theor. Chim. Acta 1965, 3, 363.

(10) Kuboyama, A.; Wada, K. Bull. Chem. Soc. Jpn. 1965, 38, 1709.

(11) Kuboyama, A.; Matsuzaki, S.; Takagi, H.; Arano, H. Bull. Chem. Soc. Jpn. 1974, 47, 1604.

(12) Srivastava, S. K.; Srivastava, S. K. Indian J. Pure Appl. Phys. 1982, $20,76$.

(13) Koenig, T.; Smith, M.; Snell, W. J. Am. Chem. Soc. 1977, 99, 6663.

(14) Schang, P.; Gleiter, R.; Rieker, A. Ber. Bunsen-Ges. Phys. Chem. 1978, 82, 629 .

(15) Bingham, R. C.; Dewar, M. J. S.; Lo, D. H. J. Am. Chem. Soc. 1975, 97, 1285 .

(16) Marks, J.; Comita, P. B.; Brauman, J. I. J. Am. Chem. Soc. 1985 , $107,3718$.

(17) Nakatsuji, H.; Hirao, K. J. Chem. Phys. 1978, 68, 2053.

(18) Nakatsuji, H. Chem. Phys. Lett. 1978, 59, 362.

(19) Nakatsuji, H. Chem. Phys. Lett. 1979, 67, 329, 334.

(20) Nakatsuji, H. Acta Chim. Hung. 1992, 129, 719.

(21) Nakatsuji, H. In Computational Chemistry, Reviews of Current

Trends; Leszczynski, J., Ed.; World Scientific: Singapore, 1997; Vol. 2.

(22) Dunning, T. H., Jr. J. Chem. Phys. 1989, 90, 1007.

(23) Dunning, T. H., Jr.; Hay, P. J. In Modern Theoretical Chemistry; Schaefer, H. F., III, Ed.; Plenum Press: New York, 1977; Vol. 3.

(24) Dunning, T. H., Jr. J. Chem. Phys. 1970, 53, 2823.

(25) Huzinaga, S.; Andzelm, J.; Klobukowski, M.; Radzio-Andzelm, E.; Sakai, Y.; Tatewaki, H. In Gaussian Basis Set for Molecular Calculations; Elsevier: Amsterdam, 1984.

(26) Macdonald, A. L.; Trotter, J. J. Chem. Soc., Perkin Trans. 21973 $4,476$.

(27) Nakatsuji, H. Chem. Phys. 1983, 75, 425.

(28) Tokita, Y.; Hasegawa, J.; Nakatsuji, H. J. Phys. Chem. A 1998, $102,1843$.

(29) Nakatsuji, H.; Hada, M.; Ehara, M.; Hasegawa, J.; Nakajima, T.; Nakai, H.; Kitao, O.; Toyota, K. SAC/SAC-CI program system (SAC-CI96) for calculating ground, excited, ionized, and electron-attached states and singlet to septet spin multiplicities, 1998.

(30) Martin, R. L.; Shirley, D. A. J. Chem. Phys. 1976, 64, 3685. 